\title{
Study on Change in Retinal Nerve Fibre Layer (RNFL) Thickness Using Optical Coherence Tomography (Spectral Domain OCT) after Trabeculectomy in Adult Patients with Glaucoma
}

\author{
S. Thakur ${ }^{1}$ \\ ${ }^{1}$ Department of Ophthalmology, Shri Shankaracharya Institute of Medical Sciences, Junwani, Bhilai, India.
}

\section{ABSTRACT}

\section{BACKGROUND}

Glaucoma is dynamic optic neuropathy with visual field defect that is related with raised intraocular pressure. Glaucoma is the second common cause for visual impairment overall which influences 67 million persons around the world, of whom around $10 \%$ or 6.6 million, are visually impaired. We wanted to evaluate changes in RNFL thickness using spectral domain OCT in trabeculectomy patients before \& after performing trabeculectomy in order to demonstrate structural improvement if any following IOP reduction in adult patients.

\section{METHODS}

Forty eyes of 30 open angle glaucoma patients attending glaucoma ophthalmology clinic at Regional Institute of Ophthalmology, Kolkata was randomly selected in accordance with the inclusion and exclusion criteria. The study got ethical committee clearance. The RNFL parameters studied were average RNFL thickness and RNFL thickness in each of four quadrants.

\section{RESULTS}

Total number of eyes in this study was 40 . Forty eyes of 30 open angle glaucoma patients were included in this study. The RNFL thickness changes in overall, trabeculectomy \& combined trabeculectomy were statistically significant. In segmental analysis, there was an increase in RNFL thickness in all quadrants, but more changes were found in nasal \& superior quadrant. The improvement is confined to the nasal quadrant \& superior quadrant, adjacent RNFL because this area tends to be least affected by the disease.

\section{CONCLUSIONS}

The study showed an increase in RNFL thickness after glaucoma filtration surgery that was correlated with IOP reduction, ages, and gender. Therefore, trabeculectomy is very helpful for IOP reduction in uncontrolled glaucoma patients with maximum medication.

\section{KEY WORDS}

RNFL (Retinal Nerve Fiber Layers), SD-OCT (Spectral-Domain Optical Coherence Tomography), IOP (Intra Ocular Pressure)
Corresponding Author:

Sangeeta Thakur,

Q. No. 636, Ward No. 51, Madhuban Nagar, Borsi,

Chhattisgarh, India.

E-mail: sangeetathakur81298@gmail.com

DOI: $10.14260 / \mathrm{jemds} / 2019 / 736$

Financial or Other Competing Interests: None.

How to Cite This Article:

Thakur S. Study on change in retinal nerve fibre layer (RNFL) thickness using optical coherence tomography (spectral domain OCT) after trabeculectomy in adult patients with glaucoma. J. Evolution Med. Dent. Sci.2019;8(45):3392-3396, DOI: 10.14260/jemds/2019/736

Submission 12-08-2019,

Peer Review 11-10-2019,

Acceptance 18-10-2019,

Published 11-11-2019. 


\section{BACKGROUND}

Glaucoma is the leading cause of irreversible blindness worldwide and is second only to cataract as the most common cause of blindness overall. The incidence of Primary open angle glaucoma was estimated 2.4 million persons per year. In India $12.8 \%$ of visual impairment are accounted to occur due to glaucoma ${ }^{2}$. In glaucoma localized or diffuse thinning of the retinal nerve fiber layer (RNFL) is reflected by loss of retinal ganglion cells, measurement of thinning of retinal nerve fiber layer has been corelated with functional damage in visual field ${ }^{3}$. Measurement of IOP is done with Goldmann applanation Tonometry. Open angle glaucoma is described by an IOP $>21 \mathrm{mmHg}$ at some stage. The OCT was first described by Huang et al in 1991.4 The latest innovation in OCT utilizes a spectrometer as a finder of OCT signal.5,6 Spectral domain OCT (SD-OCT) has benefits over the time domain OCT (TD-OCT, for example, higher axial resolution (3 to $6 \mu \mathrm{m}$ ),quicker examining pace and better reproducibility.

We wanted to measure and compare preoperative RNFL thickness and postoperative RNFL thickness using spectral domain OCT in patients who underwent trabeculectomy \& trabeculectomy with MMC.

\section{METHODS}

The study was approved by ethical committee from Medical College, Kolkata. Total number of eyes in this study was 40 . Forty eyes of 30 open angle glaucoma patients were taken in this study from 104 patients; 34 eyes underwent trabeculectomy, 6 eyes underwent trabeculectomy with intraoperative application of mitomycin c. Each subject had a comprehensive ophthalmic examination including bestcorrected visual acuity (BCVA). Stereoscopic fundus evaluation was done on the slit lamp using a 90.0-D lens. IOP was measured by Goldmann Applanation tonometry. Gonioscopy was done using the Goldmann two mirror gonioscope. RNFL changes precede the appearance of functional changes in the visual field and have greater value as early indicator of the disease. Patients were candidates for trabeculectomy if intraocular pressure was not adequately controlled on medications. Intraocular pressure was not controlled in advanced glaucoma patients and due to noncompliance. Postoperative visual acuity, IOP, slit lamp biomicroscopy, and disc evaluation was done at, week 1, 1 month, and then 3 months postoperatively. Post-op visualacuity, slit lamp biomicroscopy were done on day 1 . Visual field preoperative within 2 weeks \& postoperative 1 week, 1 month \& 3 months was assessed.

\section{Statistical Analysis}

Statistical analysis plan or any other issue relevant to research was analysed following standard statistical protocol using MATLAB software. This was an institutional based, Prospective, clinical study. For statistical analysis data were entered into a Microsoft excel spreadsheet and then analysed by SPSS 10.0.1 and Graph Pad Prism version 5. Data have been summarized as mean and standard deviation for numerical variables and percentages for categorical variables. Student's independent sample's t-test was applied to compare normally distributed numerical variables between groups, unpaired proportions were compared by Chi-square test as appropriate. Once a $t$ value is determined, a $p$-value can be found using a table of values from Student's tdistribution if the calculated p-value is below the threshold chosen for statistical significance (Usually the 0.10 , the 0.05 , or 0.01 level), then the null hypothesis is rejected. The intraocular pressure is statistically significant in different surgical procedures. All patients IOP has reduced from 28.55 $\pm 8.47 \mathrm{mmHg}$ to $14.90 \pm 4.8 \mathrm{mmHg}(\mathrm{p}<0.0001)$. In Trabeculectomy group \& combined Trabeculectomy with mmc group the IOP reduction was from $28.82 \pm 8.40 \mathrm{mmHg}$ to $15.17 \pm 5.11 \mathrm{mmHg}$ and $27.00 \pm 9.52 \mathrm{mmHg}$ to $13.33 \pm$ $2.06 \mathrm{mmHg}$ respectively $(\mathrm{p}<0.0001 \& \mathrm{P}=0.0002$ respectively).

\section{RESULTS}

The RNFL thickness changes in overall, trabeculectomy \& combined trabeculectomy were statistically significant. The mean age (mean \pm S. D. ) of patients was $51.50 \pm 9.33$ years with range 38.00-70.00 years and the median age was 49.00 years. The RNFL thickness change (mean \pm SD) was $51.04 \pm$ $17.41 \mu \mathrm{m}$ for overall RNFL in both trabeculectomy \& trabeculectomy with mmc group $(\mathrm{P}=0.1847)$. The RNFL thickness change was statistically significant in both trabeculectomy and combined procedure. We observed a mean overall RNFL thickness increase in 29 (72.5\%) of 40 eyes \& mean RNFL thickness decrease in 11 (27.5\%) of 40 eyes. But the overall RNFL thickness was significantly increased $9.08 \pm 3.50 \mu \mathrm{m}$. $(\mathrm{p}=0.1847)$. In segmental analysis, there was an increase in RNFL thickness in all quadrants, but more changes were found in nasal \& superior quadrant. The improvement is confined to the nasal quadrant \& superior quadrant, adjacent RNFL because this area tends to be least affected by the disease. Strengths of the study were quantitative measures were done. Limitations of the study: small sample size, subsequent follow up, and long-term study was essential to assess clinical significance of changes.

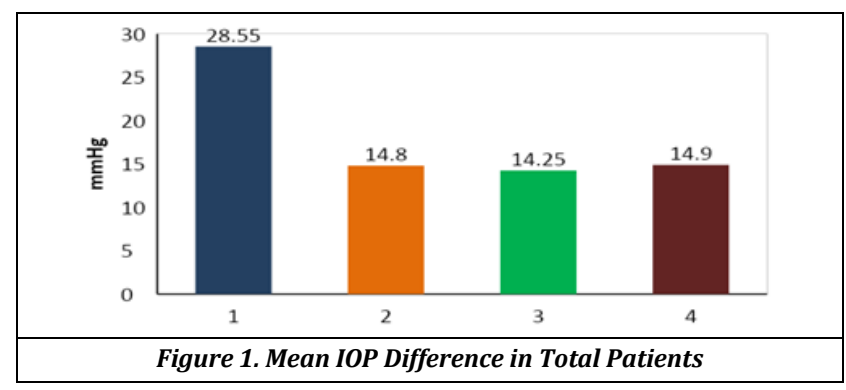

Mean IOP difference in Pre-op patients \& postop, 1 week, 1 month and 3 months follow-up in total patients was statistically significant $(\mathrm{p}<0.0001)$. Preoperative OCT images of peripapillary NFL were obtained 0 to 60 days before surgery \& postoperative images were obtained from 1 week, 1 month, 3 months after surgery. 


\begin{tabular}{|c|c|c|c|c|c|c|c|}
\hline & & No. & Mean & S. D. & Min & Max & Median \\
\hline \multirow{5}{*}{ Total } & Pre-op & 40 & 51.0495 & 17.4188 & 46.7500 & 100.5000 & 48.0000 \\
\cline { 2 - 8 } & 1 Week & 40 & 59.6913 & 22.7230 & 42.0800 & 123.0000 & 55.7950 \\
\cline { 2 - 8 } & Months & 40 & 58.6375 & 23.7086 & 45.0000 & 115.7500 & 54.8750 \\
\cline { 2 - 8 } & Months & 40 & 60.2625 & 20.9168 & 44.2500 & 112.0000 & 55.6250 \\
\hline \multirow{4}{*}{ Trabeculectomy } & Pre-op & 34 & 50.4276 & 17.9324 & 46.7500 & 100.5000 & 47.1600 \\
\cline { 2 - 8 } & 1 Week & 34 & 60.1291 & 23.9967 & 42.0800 & 123.0000 & 54.6900 \\
\cline { 2 - 7 } & 1 Months & 34 & 59.0882 & 25.2838 & 45.0000 & 115.7500 & 54.8750 \\
\cline { 2 - 7 } & 3 Months & 34 & 60.5956 & 22.1622 & 44.2500 & 112.0000 & 54.5000 \\
\hline \multirow{3}{*}{$\begin{array}{c}\text { Trabeculectomy } \\
\text { with MMC }\end{array}$} & Pre-op & 6 & 54.5733 & 15.0582 & 45.5000 & 78.0000 & 55.1200 \\
\cline { 2 - 7 } & 1 Week & 6 & 57.2100 & 14.7706 & 46.0000 & 78.0000 & 58.3900 \\
\cline { 2 - 7 } & 1 Months & 6 & 56.0833 & 12.4887 & 42.5000 & 73.5000 & 53.8750 \\
\cline { 2 - 7 } & 3 Months & 6 & 58.3750 & 12.8800 & 45.5000 & 80.0000 & 56.2500 \\
\hline Table 1. Comparison of Average RNFL in Pre-op \& Postop 1 Week, 1 \\
\end{tabular}

Table 1 summarizes the mean peripapillary RNFL thickness, a significant increase in RNFL thickness was found for the overall measurement \& in all quadrants. The RNFL thickness change (mean \pm SD) was $51.04 \pm 17.41 \mu$ m for overall RNFL in both trabeculectomy \& trabeculectomy with mmc group $(\mathrm{P}=0.1847)$. The RNFL thickness change was statistically significant in both trabeculectomy and combined procedure. We observed a mean overall RNFL thickness increase in 29 $(72.5 \%)$ of 40 eyes \& mean RNFL thickness decrease in 11 (27.5\%) of 40 eyes. But the overall RNFL thickness was significantly increased $9.08 \pm 3.50 \mu \mathrm{m}$. ( $\mathrm{p}=0.1847)$. Mean Average RNFL difference in pre-op \& postop 1 week, 1 month and 3 months follow-up in total patients was statistically significant $(\mathrm{p}=0.1847)$.
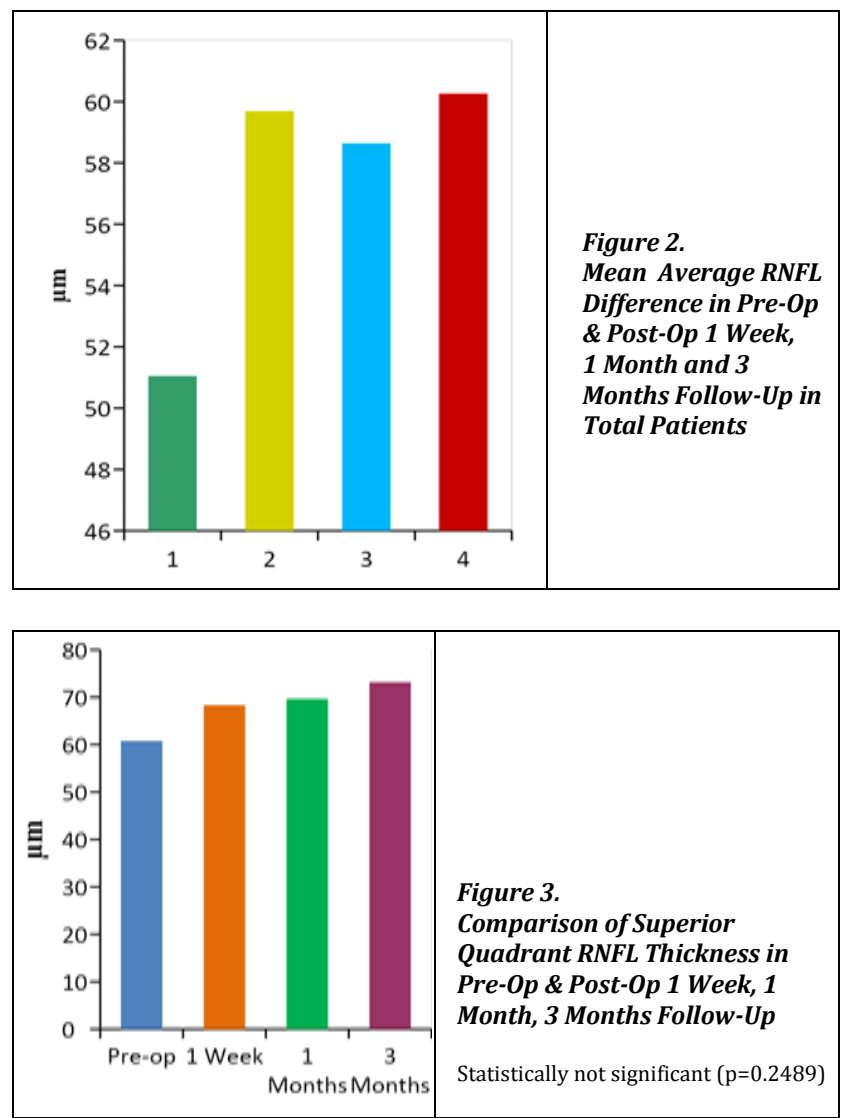

\begin{tabular}{|c|c|c|c|c|c|c|}
\hline & No. & Mean & Std. Dev. & Minimum & Maximum & Median \\
\hline Pre-op & 40 & 60.8000 & 23.4534 & 22.0000 & 125.0000 & 54.0000 \\
\hline 1 Week & 40 & 68.3250 & 28.3561 & 34.0000 & 153.0000 & 64.5000 \\
\hline 1 Months & 40 & 69.6500 & 29.4736 & 21.0000 & 161.0000 & 64.5000 \\
\hline 3 Months & 40 & 73.1750 & 28.7339 & 36.0000 & 150.0000 & 69.0000 \\
\hline & Table 2. Comparison of Superior Quadrant RNFL Thickness in \\
Pre-Op \& Post-Op 1 Week, 1 Month and 3 Months Follow-Up \\
\hline
\end{tabular}

\section{DISCUSSION}

A personal interview was conducted to determine profile, exposure to the risk factors of glaucoma, ocular trauma, past treatment for glaucoma. History was taken \& general checkup was done to rule out diabetes, anaemia \& hypertension. Slit lamp examination was done to note the status of conjunctiva, cornea, anterior chamber, iris \& lens. In recognition of the importance of bleb appearance in relation to surgical outcome and complications, a number of classification systems have been proposed to characterize bleb morphology based on slit lamp grading.7,8 Fundus examination was done. Optic disc was examined after dilating each pupil with one drop of a mixture of tropicamide $0.8 \%$ \& phenylephrine $5 \%$. A ratio of the longest vertical diameter of the cup to the longest vertical diameter of disc was estimated as the VCDR of each eye ${ }^{1}$. Visual field examination was done with automated visual field (Humphrey Field Analyzer 24-2 program) analysis. Study setting included RIO Glaucoma OPD clinic at Regional Institute of Ophthalmology, Medical College Kolkata. Timeline period was 18 months from January 2014 to august 2015. Inclusion criteria were patients attending OPD at Regional Institute of Ophthalmology, Kolkata. With Primary Open angle glaucoma with informed consent to undergo Trabeculectomy and follow up. Patients $>18$ years of age, with unsatisfactory IOP control for the status of their glaucoma while on maximum tolerable anti-glaucoma medications. Exclusion criteria were a previous history of ocular trauma. Corneal oedema, ocular disease or intraocular surgery or laser iridotomy, laser trabeculoplasty or diabetic retinopathy, uveitis, or cataract, age related macular degeneration. Cystoid macular oedema, macular dystrophy, central serous retinopathy, angle closure glaucoma.

The study design was Prospective, interventional, clinical study, sample size was Patients attending Glaucoma ophthalmology clinic at Regional Institute of Ophthalmology, Kolkata were randomly selected in accordance with the inclusion and exclusion criteria. Sampling design was Random sampling from the patients chosen as candidate for glaucoma and who give consent for trabeculectomy surgery. The RNFL thickness change (mean \pm SD) was preoperatively $51.04 \pm 17.41 \mu \mathrm{m} \&$ was $60.26 \pm 20.91 \mu \mathrm{m}$ postoperatively for overall RNFL $(\mathrm{P}=0.1847)$, Superior Average were preoperatively $60.80 \pm 23.45 \mu \mathrm{m} \& 73.17 \pm 28.73 \mu \mathrm{m}$ postoperatively $(\mathrm{p}=0.2410)$, Inferior Average were preoperatively $53.20 \pm 30.82 \mu \mathrm{m}$ and $67.82 \pm 38.37 \mu \mathrm{m}$ $(\mathrm{p}=0.2595)$ post-operatively. $(\mathrm{p}=0.2595)$ Nasal average were preoperatively $47.32 \pm 18.50 \mu \mathrm{m} \& 55.20 \pm 20.25 \mu \mathrm{m}$ postoperatively $\quad(\mathrm{p}=0.2489)$, Temporal mean were preoperatively $42.45 \pm 13.86 \mu \mathrm{m} \& 46.02 \pm 14.99 \mu \mathrm{m}$ $(\mathrm{p}=0.3365)$ in trabeculectomy and trabeculectomy with $\mathrm{mmc}$ groups.

In this study the mean deviation (dB) decibel value of Automated Perimetry (24-2 SITA STANDARD) results were preoperatively $-13.95 \pm 7.88 \mathrm{~dB} \&-10.61 \pm 5.48 \mathrm{~dB}$ postoperatively $(\mathrm{P}=0.2192)$. Percentage of sex was equally distributed in this study, 15 males \& 15 females. The intraocular pressure is statistically significant in different surgical procedures. In overall patients the intraocular pressure has reduced from $28.55 \pm 8.47 \mathrm{mmHg}$ to $14.90 \pm 4.8$ $\mathrm{mmHg}(\mathrm{p}<0.0001)$. In Trabeculectomy group \& combined 
Trabeculectomy with mmc group the IOP reduction was from $28.82 \pm 8.40 \mathrm{mmHg}$ to $15.17 \pm 5.11 \mathrm{mmHg}$ and $27.00 \pm 9.52$ $\mathrm{mmHg}$ to $13.33 \pm 2.06 \mathrm{mmHg}$ respectively $(\mathrm{p}<0.0001 \&$ $\mathrm{P}=0.0002$ respectively). Spectral- domain optical coherence tomography is a method of using low-coherence interferometry to determine the echo time delay and magnitude of backscattered light reflected off an object of interest. This method can be used to scan through the layers of a structured tissue sample such as the retina with very high axial resolution ( 3 to $15 \mu \mathrm{m}$ ), providing images demonstrating 3D structure. Because of the unique optically clear pathway through the eye, OCT has been used most extensively for imaging disorders affecting the retina.

\section{Why 3D OCT is Better than Stratus OCT}

In glaucoma patients, measurements of the RNFL reveals a high correlation of OCT and GDx devices because OCTs can measure all major retinal layers and SLP can detect nerve fibers so SLP detects comparison between the results of this devices. However, CSLO by means of HRT topography can only measure height values of the retinal surface but it cannot distinguish between different retinal layers. SD OCTs detects physical differences in the imaging methods, and it is software dependent. So measurement with SD OCTs are generally higher than with GDx VCC and GDx. In clinical glaucoma RNFL protocols and printouts of almost all OCT devices, blood vessels are not extracted from RNFL measurements. This is crucial especially if glaucoma is progressing because nerve fibers are decreasing, RNFL is decreasing, and the ratio between RNFL and blood vessels is markedly changing. Although OCT technology can differentiate between RNFL and blood vessels. (e. g.2 mouse clicks in Topcon SD-OCT 2000) this helpful feature is not included in standard OCT RNFL software protocols. This is an advantage of SLP because blood vessels have no birefringent properties and therefore, they do not disturb GDx RNFL measurements.

\section{Limbus-Based v/s Fornix-Based Trabeculectomy}

IOP control is comparable with both techniques.

\section{Fornix - Based}

Advantages: intraoperative benefits and better bleb morphology Disadvantage: increased incidence of early bleb leaks.

\section{Limbus - Based}

Advantage: more secure wound closure. Disadvantages: late complications of leaks \& infection

Caution should be taken in patients on anticoagulative therapy as the bleeding complications are increased. If anticoagulants cannot be stopped, the patient can be started on Lovenox (Enoxaparin sodium injection) temporarily. Extensive scarring of superior conjunctiva can preclude the creation of a conjunctival flap, inferior tube surgery or cyclodestructive procedures may provide better choices.

Aydin et al (9) used a non-commercial, prototype OCT device to evaluate RNFL thickness change following trabeculectomy, and reported a significant increase. The differences were more marked in the group that had the cataract surgery also. Segmental analysis found a significant increase in RNFL thickness in the nasal, superior, and temporal quadrants. Irak et al reported significant changes in several optic disc parameters on the HRT 4.5 months after surgery, which had a significant association with the IOP reduction. The limitations of our study were small sample size in each subgroup. We have taken only the mean RNFL thickness and mean Retinal Sensitivity, but no sectoral wise comparison is taken into account. Another limitation of this study is we did not evaluate the Optic nerve head topography which shows to have a greater diagnostic ability in discriminating glaucomatous from non-glaucomatous eyes.

\section{CONCLUSIONS}

The study showed an increase in RNFL thickness after glaucoma filtration surgery that was correlated with IOP reduction, ages, and gender. Therefore, trabeculectomy is very helpful for IOP reduction in uncontrolled glaucoma patients with maximum medication. More elderly patients and patients with advanced glaucomatous damage were included in this study and this hinders the generalization in a specific group of patients.

\section{ACKNOWLEDGEMENT}

Authors acknowledge their teachers, post graduate students, nursing and paramedical staff at the Department of Ophthalmology, Regional Institute of Ophthalmology, Medical College, Kolkata. Authors thank all the patients who participated in this study.

\section{REFERENCES}

[1] Quigley HA, Broman AT. The number of people with glaucoma worldwide in 2010 and 2020.Br J Ophthalmol 2006;90(3):262-7.

[2] Garway-Heath DF, Poinoosawmy D, Fitzke FW, et al. Mapping the visual field to the optic disc in normal tension glaucoma eyes. Ophthalmology 2000;107(10):1809-15

[3] Reus NJ, Lemij HG. The relationship between standard automated perimetry and GDx VCC measurements. Invest Ophthalmol Vis Sci 2004;45(3):840-5.

[4] Huang D, Swanson EA, Lin CP, et al. Optical coherence tomography. Science 1991;254(5035):1178-81.

[5] Sakata LM, Deleon-Ortega J, Sakata V, et al. Optical coherence tomography of the retina and optic nerve - a review. Clinical Experiment Ophthalmol 2009;37(1):909.

[6] Chen TC Spectral domain optical coherence tomography in glaucoma: qualitative and quantitative analysis of the optic nerve head and retinal nerve fiber layer (an AOS thesis). Trans Am Ophthalmol Soc 2009;107:254-81. 
[7] Cantor LB, Mantravadi A, WuDunn D. et al. Morphologic classification of filtering blebs after glaucoma filtration surgery: the Indiana Bleb Appearance Grading Scale. J Glaucoma 2003;12(3):266-71.

[8] Wells AP, Crowston JG, Marks J, et al. A pilot study of a system for grading of drainage blebs after glaucoma surgery. J Glaucoma 2004;13(6):454-60.
[9] Aydin A, Wollstein G, Price LL, et al. Optical coherence tomography assessment of retinal nerve fiber layer thickness changes after glaucoma surgery. Ophthalmology 2003;110(8):1506-11. 